\title{
An uncommon cause of back pain in pregnancy
}

\author{
R Vadivelu, T P Green, R Bhatt
}

Postgrad Med J 2005;81:65-67. doi: 10.1136/pgmj.2003.015370

Back pain is a major cause of disability worldwide. A case of a 29 year old Caucasian woman with low back pain secondary to an uncommon benign condition called osteitis condensans ilii is presented and the literature is reviewed. Limited information in the literature combined with poor awareness across the specialty and among the primary care physicians leads to an extensive investigation and misdiagnosis of this benign condition. This report emphasis the salient features and the differential diagnosis in diagnosing and managing this rare condition.

$\mathrm{B}$ ackache is a common symptom in women in pregnancy and during the postpartum period. Ligamentous laxity and extra mechanical stress have been attributed as the main cause for back pain during pregnancy. ${ }^{12}$ In a small group of patients the persistent pain during the postpartum period may be secondary to osteitis condensans ilii (OCI). This case report highlights the salient features and discusses the differential diagnosis and management of this benign condition.

\section{CASE REPORT}

A 29 year old Caucasian woman presented with a two year history of low back pain. The symptoms started during the postpartum period of her second pregnancy. The pain was gradual in onset and then became constant. She described the pain as being across her lower back and buttocks and it occasionally radiated down the back of her legs but not beyond the knee. Coughing or sneezing did not aggravate the pain but she generally experienced discomfort in most activities, particularly if she crouched or bent down. She had never complained of any numbness or "pins and needles" nor weakness in her legs. She had no history suggestive of morning stiffness or other joint involvement. She had no family history of joint disorders. There was no relief from analgesics and anti-inflammatories.

Clinically, her body mass index was 32 . She had a slightly increased lumbar lordosis and her gait was normal. She had no wasting of muscles. She was able to do a tiptoe and heel walking without any difficulty. Flexion, extension, rotation, and lateral bending of her lumbar spine were normal. On palpation, she had no specific bony or paraspinal muscle tenderness. She could demonstrate a straight leg raise up to 90 degrees on both sides and sciatic stretch test and femoral stretch test were negative. Power, tone, reflexes, and sensation were normal bilaterally. The FABER test (FlexionABduction External Rotation) or the figure of four test provoked the pain in her buttocks. This test is also known as Patrick's test and is performed with the patient supine on the examination table. The limb to be examined is guided into figure of four position with the ipsilateral ankle resting across the contralateral thigh. The examiner then presses downwards on the ipsilateral knee with one hand while providing counterpressure with the other hand on the contralateral anterior superior iliac spine. This manoeuvre tends to stress the sacroiliac joint on the side being tested. Sacroiliac joint pathology typically produces posterior hip pain and in an arthritic hip pain is usually felt anteriorly in the groin. Table 1 shows her baseline blood investigations, which were all normal. An anteroposterior plain radiograph of her lumbar spine is shown in fig 1 .

The anteroposterior plain radiograph of the lumbar spine shows bilateral symmetrical triangular shaped sclerosis around the lower part of the sacroiliac joints. The sclerosis is mainly affecting the iliac side of the sacroiliac joints. There is no evidence of narrowing or irregularity in the joint space on both sides. These salient features are diagnostic of OCI, which is a benign self limiting condition. It is important to recognise this entity as it can be potentially confused with other disorders, as mentioned below in the discussion.

Treatment in OCI is conservative with physiotherapy and analgesia as required. Our patient had a course of physiotherapy and made a satisfactory recovery.

\section{DISCUSSION}

Backache is a common symptom in women in pregnancy and during the postpartum period. Increased ligamentous laxity secondary to the release of the hormone relaxin, increase in

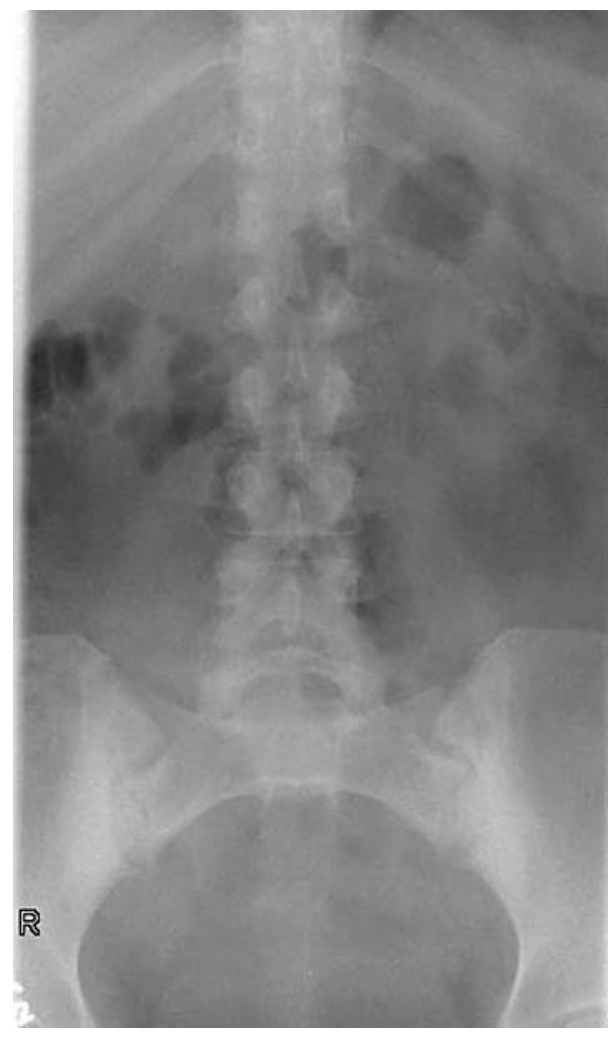

Figure 1 Anteroposterior view of lumbar spine. 
Table 1 Baseline blood investigation with normal reference range

\begin{tabular}{|c|c|c|}
\hline & Result & Reference range \\
\hline \multicolumn{3}{|l|}{ Haematology } \\
\hline Haemoglobin (g/l) & 153 & $115-165$ \\
\hline White cell count $\left(\times 10^{9} / \mathrm{l}\right)$ & 11.2 & $4.0-11.0$ \\
\hline Red blood count $\left(\times 10^{9} / \mathrm{I}\right)$ & 4.77 & $3.9-5.6$ \\
\hline Packed cell volume & 0.424 & $0.37-0.47$ \\
\hline Mean cell volume (fl) & 89 & $80-99$ \\
\hline Mean cell haemoglobin (pg) & 32.1 & $27-32$ \\
\hline Platelet count $\left(\times 10^{9} / \mathrm{l}\right)$ & 241 & $150-400$ \\
\hline Neutrophil count $\left(\times 10^{9} / 1\right)$ & 7.70 & $2-7.5$ \\
\hline Lymphocyte count $\left(\times 10^{9} / \mathrm{I}\right)$ & 2.40 & $1.5-4$ \\
\hline Monocyte count $\left(\times 10^{9} / \mathrm{II}\right)$ & 0.60 & $0.2-0.8$ \\
\hline Eosinophil count $\left(\times 10^{9} / \mathrm{l}\right)$ & 0.50 & $0.04-0.4$ \\
\hline Basophil count $\left(\times 10^{9} / \mathrm{I}\right)$ & 0.10 & $0.01-0.1$ \\
\hline Plasma viscosity (cp) & 1.72 & $1.5-1.72$ \\
\hline \multicolumn{3}{|l|}{ Biochemistry } \\
\hline Sodium (mmol/l) & 137 & $133-144$ \\
\hline Potassium (mmol/l) & 4.5 & $3.3-5.3$ \\
\hline Urea (mmol/l) & 3.1 & $2.5-6.5$ \\
\hline Creatinine $(\mathrm{mmol} / \mathrm{l})$ & 80 & $60-120$ \\
\hline Total bilirubin ( $\mu \mathrm{mol} / \mathrm{l})$ & 9 & $3-17$ \\
\hline Albumin (g/l) & 49 & $35-55$ \\
\hline Adjusted calcium (mmol/l) & 2.53 & $2.10-2.60$ \\
\hline Inorganic phosphate (mmol/l) & 1.01 & $0.80-1.40$ \\
\hline Alkaline phosphatase (IU/I) & 70 & $40-130$ \\
\hline C-reactive protein (mg/l) & $<5$ & $0-10$ \\
\hline
\end{tabular}

body weight due to weight gain, and forward weight bearing are the major factors responsible for back pain in pregnancy. Extra mechanical stresses and nerve and vascular impingement from mechanical pressure have also been shown to contribute to the back pain. ${ }^{3}$

A study among the Swedish women between the ages of 38 and 64 showed that two thirds of them had experienced back pain at some time, but only a small group reported that it started during pregnancy. ${ }^{3}{ }^{4}$ Current reports show that the back pain experienced during pregnancy can be intense and may persist after pregnancy. One third of women who experience pain during the postpartum period have reported that their back pain affects their day-to-day activities, and in about $10 \%$ of them it prevents them from working. ${ }^{3}$

Most back pain settles after pregnancy with simple measures. In a small group of patients back pain may persist and can hinder their normal daily activities. Extensive investigations including magnetic resonance imaging not only increase anxiety but are of little benefit. Correct diagnosis and reassurance with simple treatment measures can yield satisfactory results in this group of patients. Primary care physicians and other specialists who deal with patients suffering from back pain need to be aware of these uncommon benign disorders, of which OCI is one.

Current orthopaedic and rheumatology literature reveals very little information regarding OCI and there is hardly any mention of it in the publications commonly referred to by primary care physicians for their everyday practice.

OCI is a benign condition typically seen after pregnancy and is not associated with any inflammatory arthritis. ${ }^{56}$ It is essentially a radiological diagnosis. The iliac bone adjacent to the sacroiliac joint is predominantly affected and usually, although not invariably, it is a bilateral and relatively symmetric process. It is more common in women of childbearing age group. Rarely, nulliparous women and men may be affected and it can be unilateral. The radiological appearance in OCI is characteristic with well defined triangular sclerosis on the iliac aspect of the sacroiliac joint. The bony eburnation involves the inferior portion of the bone and the apex of the sclerosis can extend up to the auricular portion of the ilium.
Table 2 Differences between $\mathrm{OCl}$ and ankylosing spondylitis

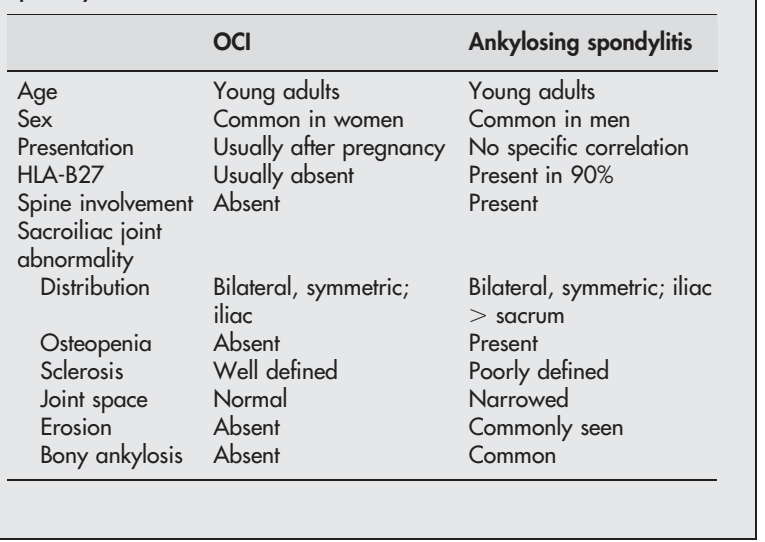

In OCI, the joint space is preserved and the articular margins are intact, unlike in other forms of sacroiliitis. These radiological features may vary in extent and may even resolve with time. ${ }^{7}$ The radiological features and the clinical history are diagnostic of OCI, but other differential diagnoses include seronegative spondyloarthropathies, renal osteodystrophy, lymphoma, Paget's disease, and primary hyperparathyroidism. Normal blood parameters and seronegativity exclude the majority of these disorders. Polyarthralgia in peripheral joints have been noted in a small group of patients with OCI, although significant inflammatory articular findings are generally absent.

The aetiology of OCI is not clear and many theories have been postulated. It is suggested that the increased mechanical stress across the sacroiliac joint coupled with increased vascularity during pregnancy leads to the changes seen on the iliac bone. However, OCI seen in nulliparous women and in men is not consistent with this theory, although it is assumed that mechanical stress from a different causation may be operational in this group of patients. Some authors have postulated that OCI and ankylosing spondylitis may be inter-related; however the aetiopathology of ankylosing spondylitis is different from OCI (table 2). ${ }^{8}$ Singal et al in their comparative studies between OCI and ankylosing spondylitis in female patients have concluded that OCI is not a variant of ankylosing spondylitis. ${ }^{910}$

To conclude: OCI is a benign self limiting condition seen after pregnancy in young women with low back pain. It is important to recognise this entity as it may potentially be confused with sacroiliitis from other disorders like ankylosing spondylitis and inflammatory arthritis. Conservative treatment with analgesics and physiotherapy are the mainstay of management in OCI.

\section{Authors' affiliations}

R Vadivelu, T P Green, R Bhatt, University Hospitals of Leicester NHS Trust, Leicester General Hospital, Leicester, UK

Correspondence to: Mr R Vadivelu, Department of Orthopaedics, Leicester General Hospital, Gwendolen Road, Leicester LE5 4PW, UK; rvadivelu@yahoo.com

Submitted 24 September 2003

Accepted 29 March 2004

\section{REFERENCES}

1 Ostgaard HC, Andersson GBJ, Schultz AB, et al. Influence of some biomechanical factors on low back pain in pregnancy. Spine 1993;18:61-5. 2 Maclennan AH, Nicolson R, Green RC, et al. Serum relaxin and pelvic pain of pregnancy. Lancet 1986;ii:243-5. 
3 Ostgaard HC, Andersson GBJ, Karlsson K. Prevalence of back pain in pregnancy. Spine 1991;16:549-52.

4 Svensson HO, Andersson GBJ, Hagstad A, et al. The relationship of low back pain to pregnancy and gynecological factors. Spine 1990;15:371-5.

5 Thompson M. Osteitis condensans ilii and its difference from anklylosing spondylitis. Ann Rheum Dis 1954;13:147.

6 Resnick D. Disorders of other endocrine glands and of pregnancy. In: Resnick D, ed. Diagnosis of bone and joint disorders. Philadelphia: W B Saunders, 1995:2089-92.
7 Numaguchi Y. Osteitis condensans ilii, including its resolution. Radiology 1971;98:1-8.

8 de Bosset P, Gordon DA, Smythe HA, et al. Comparison of osteitis condensans ilii and ankylosing spondylitis in female patients: clinical, radiological and HLA typing characteristics. J Chronic Dis 1978;31:171-81.

9 Singal DP, de Bosset $P$, Gordon DA, et al. HLA antigens in osteitis condensans ilii and ankylosing spondylitis. J Rheumatol Suppl 1977;3:105-8.

10 Hamill CL. Spinal and pelvic manifestation of rheumatoid arthritis and the spondyloarthropathies. In: Dee R, Hurst LC, Gruber MA, et al, eds.

Principles of orthopedic practice. New York: McGraw Hill, 1997:1373-9.

\section{IMAGES IN MEDICINE}

\section{Cardiac tamponade}

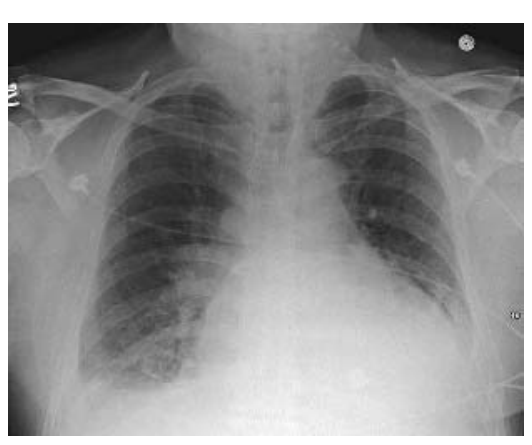

Figure 1 Chest radiograph demonstrating blunting of both costophrenic angles and an enlarged pericardial silhovette.

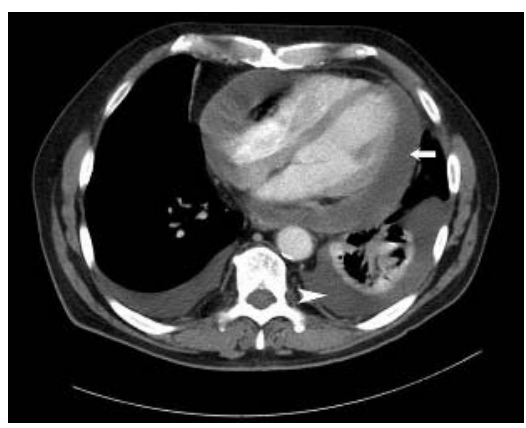

Figure 2 Computed tomography: arrow demonstrates pericardial fluid collection; arrowhead demonstrates pleural fluid collection.

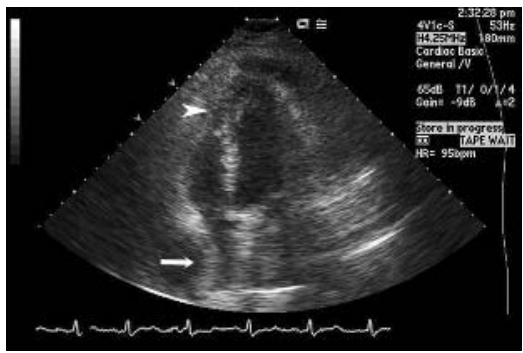

Figure 3 Two dimensional echocardiogram: arrow demonstrates right end diastolic atrial collapse; arrowhead demonstrates right end diastolic ventricular collapse, consistent with tamponade physiology.
4 68 year old man was seen in clinic for progressive fatigue, dyspnoea on exertion, and cough which was progressive over one week. He denied any chest pain. He was being treated on a protocol for metastatic renal cell carcinoma. The patient initially had a chest radiograph taken, which revealed blunting of both costophrenic angles and an enlarged pericardial silhouette (fig 1). To evaluate the pericardial fluid and to rule out progressive disease computed tomography of the chest was performed. This demonstrated bilateral pleural effusions (arrowhead) and a moderate pericardial fluid collection (arrow) with suggestion of compression of the cardiac contours (fig 2). The patient underwent an urgent transthoracic two dimensional echocardiogram, which showed a moderate circumferential pericardial effusion and significant end diastolic right atrial (arrow) and ventricular (arrowhead) collapse consistent with cardiac tamponade physiology (fig 3). The patient was taken to the operating room, where an emergent subxiphoid pericardiostomy was performed.

Five hundreds millilitres of bloody fluid under pressure were evacuated without complication. The patient's symptoms resolved and he was discharged to home without recurrence of the circumferential pericardial effusion.

J Farma, D Nguyen Surgical Branch, National Cancer Institute, Bethesda, MD 20892, USA; farmaj@mail.nih.gov 